\title{
Comparativa de los temas tratados por los principales partidos políticos y sus candidatos en Twitter y sus programas electorales. Análisis de las elecciones generales de 2016 en España
}

\section{Leire Iturregui Mardaras ${ }^{1}$ María José Cantalapiedra González ${ }^{2}$ Angeriñe Elorriaga IIlera ${ }^{3}$ Bruno Caneda Portela ${ }^{4}$}

Recibido: 23/01/2020

Aprobado por pares: 15/04/2020
Enviado a pares: $28 / 01 / 2020$

Aceptado: 07/07/2020

DOI: 10.5294/pacla.2021.24.4.5

Para citar este artículo / to reference this article / para citar este artigo Iturregui, L., Cantalapiedra, M. J., Elorriaga, A. y Caneda, B. (2021). Comparativa de los temas tratados por los principales partidos políticos y sus candidatos en Twitter y sus programas electorales. Análisis de las elecciones generales de 2016 en España. Palabra Clave, 24(4), e2445. https://doi.org/10.5294/pacla.2021.24.4.5

\section{Resumen}

El presente estudio analiza el uso que los principales partidos políticos hacen de la red social Twitter en la campaña electoral correspondiente a las elecciones españolas generales del año 2016. Para ello se han seleccionado las cuentas de Twitter de los cuatro partidos que alcanzaron un $86 \%$ del porcentaje total de votos: el Partido Popular (PP), el Partido Socialista Obrero Español (PSOE), Unidas Podemos (UP) y Ciudadanos (C's). Ade-

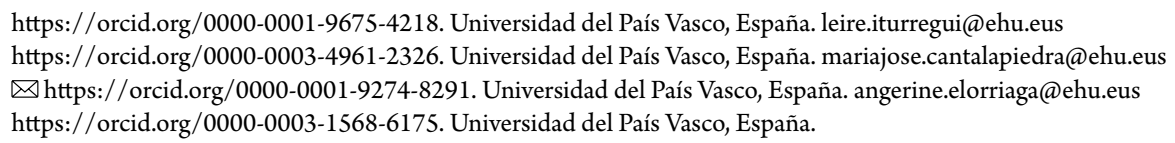


más, también se han tenido en cuenta las publicaciones de los respectivos candidatos de cada partido a la presidencia del gobierno y se han analizado los temas que tratan en esas publicaciones en Twitter, para compararlos con los temas que proponen en sus programas electorales. Los resultados de los 4.329 trinos (tweets) analizados demuestran la falta de coherencia entre los temas abordados en los trinos de partidos y candidatos, así como la ausencia de proporcionalidad en la importancia otorgada a las distintas cuestiones en el programa electoral. También se concluye que la modernidad adjudicada a los partidos de nueva creación no tiene como consecuencia una mayor actividad en la red social Twitter.

\section{Palabras clave (Fuente: tesauro de la Unesco)}

Campaña electoral; comunicación política; elecciones; España; medios sociales; Twitter. 


\section{Comparing the Topics Covered by Main Political Parties and Their Candidates on Twitter and Their Party Platforms. Analysis of the 2016 General Elections in Spain}

\section{Abstract}

The present study discusses how the main political parties used Twitter during the electoral campaign in the Spanish general elections of 2016. For this purpose, we selected the Twitter accounts of the four parties that reached $86 \%$ of the total votes: Partido Popular (PP), Partido Socialista Obrero Español (PSOE), Unidas Podemos (UP) y Ciudadanos (C's).. The tweets of each party's candidate for the presidency have also been considered, comparing the topics they deal with in those tweets with their party platforms. The results of the 4,329 tweets analyzed demonstrate a lack of coherence between parties and their candidates and a lack of proportionality in the importance given to different issues in the party platform. We conclude that the modernity attributed to newly created parties does not result in increased activity on Twitter.

\section{Keywords (Source: Unesco Thesaurus)}

Electoral campaign; political communication; elections; Spain; social media; Twitter. 


\section{Comparação dos temas tratados pelos principais partidos políticos e seus candidatos no Twitter e seus programas eleitorais. Análise das eleições gerais de 2016 na Espanha}

\section{Resumo}

Neste estudo, é analisado o uso que os principais partidos políticos fazem da rede social Twitter na campanha eleitoral correspondente às eleições gerais espanholas de 2016. Para isso, foram selecionadas as contas do Twitter dos quatro partidos que atingiram $86 \%$ do total de votos: o Partido Popular (PP), o Partido Socialista Obrero Español (PSOE), Unidas Podemos (UP) e Ciudadanos (Cs). Além disso, foram consideradas publicações dos respectivos candidatos à presidência do governo de cada partido e foram analisados os temas que tratam nessas publicações no Twitter, para compará-los com os temas que propõem em seus programas eleitorais. Os resultados de 4.329 tweets analisados demonstram a falta de coerência entre os temas abordados nos tweets de partidos e candidatos, bem como a ausência de proporcionalidade na importância dada às diferentes questões no programa eleitoral. Também se conclui que a modernidade conferida aos partidos de nova criação não tem como consequência uma maior atividade na rede social Twitter.

\section{Palavras-chave (Fonte: tesauro da Unesco)}

Campanha eleitoral; comunicação política; eleições; Espanha; meios sociais; Twitter. 
La comunicación política en línea (online) empezó a cobrar importancia a inicios del siglo XXI, al punto que la forma de hacer campañas electorales cambió notablemente, combinándose las formas de campaña tradicional con las nuevas formas de campaña derivadas del uso de internet, la web 2.0 y las redes sociales. A partir de los años sesenta, políticos, sociólogos y comunicólogos comenzaron a sugerir una perspectiva de análisis que tuviera en cuenta al individuo en sociedad. Las investigaciones realizadas en esa década y la siguiente tuvieron en cuenta, además de las condiciones psicológicas de quienes votaban, elementos institucionales, como el tipo de sistema electoral. Esta fase de investigación se llamó "sistémicoinstitucional”. Y si en los años ochenta y noventa las campañas electorales se estudiaron siguiendo una "perspectiva cultural", desde hace aproximadamente 15 años se puede observar cómo los partidos políticos tradicionales y los partidos políticos de nueva creación empiezan a considerar los beneficios potenciales que puede tener la realización de campañas electorales en línea (Anduiza, 2009).

A partir de 2004, los partidos políticos y los gobiernos se dan cuenta de las potencialidades de la Web 2.0 y se aprovechan de ellas para, por ejemplo, conseguir la mayor cantidad de información posible sobre la ciudadanía o trasladarle a ella determinadas informaciones a través de internet. De esta forma, internet se considera una herramienta que brinda a las democracias nuevas formas de relación entre representantes de la política y la ciudadanía, entre políticos diferentes e incluso entre políticos y partidos. Autores como Gibson y Römmele (2008) destacan en sus investigaciones una serie de cambios derivados del uso de esas aplicaciones, que se relacionan con: el cambio en la distribución del mensaje -que pasa de una distribución vertical a una horizontal-, el aumento de medios de transmisión de información y el cambio en los canales de comunicación. Múltiples autores consideran que el primer político a nivel mundial que consiguió explotar de manera satisfactoria y exitosa todos los recursos que la web 2.0 ofrece fue Barack Obama. Sin embargo, autores como José Eduardo Jorge (2014) opinan que Obama, a su vez, fue inspirado por Ségolène Royal, la candidata socialista francesa que en 2007 perdió las elecciones contra $\mathrm{Ni}$ colas Sarkozy en la segunda vuelta. Según Hendricks y Denton (2010), las 
elecciones estadounidenses de 2008 establecieron un nuevo modelo de comunicación entre los candidatos políticos y el electorado.

En España, para Mancera y Pano (2014), fue en las elecciones de 2011 cuando los partidos tomaron conciencia de la importancia de las redes sociales en las campañas electorales y los principales partidos españoles buscaron utilizarlas de la misma forma que Obama, sobre todo Twitter, aunque también se buscó hacerlo por otras redes sociales. Por esa razón, abrieron cuentas en esas redes, con la pretensión de comunicarse con sus votantes potenciales o con cualquier persona que los siguiese por dichas redes. Hasta las elecciones locales y autonómicas de 2007 no se introdujeron las novedades implantadas en la comunicación política en las campañas que estaban teniendo lugar ese mismo año en Francia y en Estados Unidos, y el cambio más grande en las campañas electorales en España respecto al uso de internet (Pérez et al., 2013) y sus herramientas se produjo en las elecciones generales de 2008, con la plena implantación de aspectos propios de la web 2.0.

El presente trabajo estudia el uso de la red social Twitter por parte de los partidos políticos y sus candidatos en un contexto muy peculiar para la política en España, cuando se llama a las urnas a la ciudadanía para elecciones generales tres veces en cuatro años (de 2015 a 2019). En ese escenario político, se propone un análisis de la campaña electoral correspondiente a las elecciones generales españolas del año 2016, con la intención de conocer la tendencia de uso de Twitter. Pero la aportación más significativa que propone esta investigación, respecto a otras realizadas anteriormente, es que se indaga sobre si las cuestiones presentes en el programa electoral de cada partido tienen la misma presencia en los mensajes que lanzan a través de Twitter. Hay diversos autores que afirman que la actividad en Twitter aumenta en época electoral y otorgan a esta red social la función dominante de la difusión de información sobre actos de campaña (Jackson y Lilleker, 2011). Siendo una de las características de Twitter la bidireccionalidad, varias investigaciones (Alonso, Miquel y Casero, 2016) han constatado que los partidos políticos no explotan esta capacidad. Campos (2017) presenta un panorama general de la investigación de Twitter en comunicación polí- 
tica y analiza las principales tendencias, pero no encontramos trabajos que comparen los temas de los programas electorales de los partidos políticos y su presencia en sus mensajes en Twitter.

En esa línea, los objetivos específicos de la presente investigación son: 1) descubrir y comparar la actividad que cada partido político y candidato a la presidencia realizó en Twitter en la campaña de las elecciones generales de 2016;2) averiguar si las cuestiones presentes en el programa electoral de cada partido y candidato tuvieron la misma presencia en Twitter y precisar qué cuestiones o temas tuvieron mayor relevancia en sus cuentas de Twitter; 3 ) investigar la relación entre los trinos (tweets) emitidos por las cuentas de los candidatos y las de los partidos, si tratan los mismos temas o si, por el contrario, se centran en cuestiones diferentes.

\section{El uso de Twitter en la política}

Tal y como se mencionó anteriormente, el primer político en utilizar de manera exitosa las herramientas de la web 2.0, como Twitter, fue Obama. Desde entonces mucho se ha estudiado en diferentes ámbitos el uso de esta red social en política. Jungherr (2016) realizó una revisión de 127 estudios relacionados con la comunicación electoral en Twitter y propone los usos de Twitter para los partidos, candidatos y públicos. También expone métodos y diseños para la correcta extracción de la información. Según Dader (2009), los partidos españoles empezaron a realizar una "explotación demasiado tardía e indecisa de recursos”. En el año 2008, el Partido Socialista Obrero Español (PSOE) contaba por lo menos con ocho grupos de apoyo en Facebook, que acumulaban 5.730 seguidores, siendo Zapatero el líder del grupo más numeroso, con 2.300 miembros (Peytibi, Rodríguez y Gutiérrez, 2008, p. 31). Por otro lado, el Partido Popular disponía de al menos seis grupos de apoyo, que contaban con 9.304 miembros (Dader, 2009), además del propio perfil de Rajoy, con 5.000 amigos (Peytibi et al., 2008). García y Zugasti (2014) en sus investigaciones también consideran que en España la irrupción de las herramientas derivadas de la web 2.0 y de Twitter en concreto se sitúa en 2011. En estas elecciones, el candidato español socialista Alfredo Pérez Rubalcaba abrió su perfil un día después de que asumiera su candidatura en el Comité Federal del PSOE y se adelan- 
tó a Mariano Rajoy, candidato del partido de la oposición (Partido Popular), que lo hizo dos meses más tarde. La gestión de dichas cuentas recaía en ambos equipos de campaña (García y Zugasti, 2014).

Castillejo y Sémova (2012) observan que el uso de Twitter hecho por el PP y PSOE en 2011, en general, fue para informar sobre actividades electorales o para "retuitear" información. La presencia de propuestas de ambos partidos era bastante escasa y, en términos generales, no se hizo un uso adecuado de Twitter, e incluso se cometieron errores que disgustaron a muchos usuarios. Sampietro y Valera (2015) investigaron también el uso de herramientas web 2.0 y concluyeron que, en el caso del estado español, no se las usa para la conexión directa con los votantes. Los partidos estatales se han centrado en trasladar las prácticas habituales de las campañas desconectadas de internet (offline) a las campañas en línea, olvidando el poder que estas herramientas nos ofrecen para la creación de una pedagogía política y para el desarrollo de temas de debate entre la ciudadanía. Sin embargo, Congosto (2015) afirma que en las elecciones europeas de 2014 hubo un partido que sí supo utilizar positivamente las redes sociales y fue Unidas Podemos, agrupación de reciente creación que se presentaba por primera vez a unas elecciones y que obtuvo unos resultados muy positivos e inesperados. Esta formación política fue la que más actividad desplegó en redes sociales y sus mensajes en Twitter fueron los que más difusión tuvieron. Encontramos, por lo tanto, la primera evidencia de que el uso de las redes sociales marca diferencias entre los partidos tradicionales y los de nueva creación en España.

López Meri et al. (2016) también investigaron sobre la manera de comunicarse en Twitter de los políticos catalanes en el periodo de campaña electoral. En dicho trabajo se trató de evaluar si los candidatos de los partidos utilizaron Twitter simplemente como altavoz electoral o si, por el contrario, lo usaron para empatizar con los y las votantes y así hacer que estos tuvieran una imagen de ellos más cercana. Tras el análisis realizado, la investigación concluyó que las cuentas de los partidos hacen un uso básicamente institucional de Twitter. Por otro lado, cabe destacar que las cuentas de los candidatos, según López Meri et al. (2016), sí tratan de acercarse a los votantes, pero lo hacen moderadamente, mostran- 
do, por ejemplo, muchas imágenes o elementos multimedia de su día a día, para hacerse ver como personas del común. Ruiz Soler (2017) considera que el análisis de las redes sociales es actualmente muy importante, ya que la cantidad de información disponible en las mismas está alcanzando unos niveles que no se habían contemplado nunca antes. Twitter se está convirtiendo en una fuente indispensable de macrodatos (big data), concretamente de macrodatos sociales (social big data) para los investigadores que tienen la intención de implementar este tipo de datos masivos a sus proyectos de investigación. Twitter es útil para los partidos políticos, como "fuente de información que permite segmentar a los usuarios, descubrir cómo los ciudadanos participan en el debate político y cómo se agrupan por afinidad ideológica" (Congosto, Fernández y Moro, 2011) y, además, porque facilita la creación de nuevos perfiles profesionales, relacionados con el análisis de estos datos masivos en redes sociales (Elorriaga, Merchan y Vink, 2018).

En un contexto comunicativo en el que se apunta a la desintermediación periodística (Iturregui, Gutiérrez y Cantalapiedra, 2020), Giansante (2015) destaca que Twitter brinda a los políticos la posibilidad de aproximarse e interactuar con los electores, "seguir aquello que se dice sobre un candidato o una organización" y responderles "en tiempo real", por lo que, pese a tratarse de un medio social no predominante, "constituye una herramienta de gestión de la reputación y de creación del consenso político sin igual". Por otro lado, esta red se caracteriza también por "su capacidad para condensar mensajes de todo tipo en un espacio breve, así como el carácter público de dichos mensajes y su posible difusión a gran escala", elementos que "convierten a Twitter en un instrumento idóneo para que los líderes de opinión traten de marcar la agenda del ámbito en el que operan" (López et al., 2015). Mancera y Pano (2014) también trataron la reciprocidad en Twitter. Para estas autoras las conexiones en esta red social se basan en el seguimiento, sin que sea obligatoria la reciprocidad, como ya se ha señalado; es decir, no tienes por que seguir a quien te sigue y viceversa.

López, Marcos y Casero (2017) también estudiaron las elecciones de 2016 en España y mostraron que se detectaba un bajo nivel de personalización en el contenido. Además, al apreciar diferencias entre los partidos ya establecidos y tradicionales (PSOE y PP) y los de nueva creación (Uni- 
das Podemos y Ciudadanos) hallaron que "los representantes de la nueva política cuentan con un discurso centrado en la idea del cambio y la regeneración política frente a los partidos y líderes tradicionales que destacan por una mayor referencia a los valores del partido". Existen más artículos que analizan el uso de Twitter por un partido de izquierda o de derechas. Encontramos así otro interesante artículo en el que se analiza el cambio estratégico de las campañas electorales de EH Bildu -partido vasco independentista y de izquierda- desde 2011 hasta las elecciones autonómicas de septiembre de 2016 (Orbegozo, Iturbe y González, 2017). La investigación determina que el nuevo enfoque implementado en las redes sociales y la profesionalización de este fue fruto de un diagnóstico en el que se sentaron las bases y los principios para la nueva narrativa digital.

En la esfera internacional también existen múltiples trabajos que analizan el uso de Twitter en política. Un estudio realizado durante las elecciones presidenciales de los EE. UU. en 2012 (Conway, Kenski y Wang, 2012) analiza la presencia de los candidatos en la red social, la frecuencia de publicaciones y su efecto en el aumento de seguidores en Twitter. Otra investigación, realizada en el Reino Unido durante las elecciones de 2010 (Broersma y Graham, 2013), pretende crear una tipología recogiendo los diferentes modos como los candidatos se comportan en Twitter: tipo de trino, interacciones, funciones y temas. Los resultados concluyen que los políticos británicos usan Twitter como herramienta de comunicación unidireccional, por la carencia de interacciones que se perciben en sus actividades. Kruikemeier (2014), por su lado, investiga el contenido en Twitter durante la campaña electoral de 2014 en Holanda y la relación entre el contenido generado y los votos obtenidos. Las conclusiones señalan que los candidatos que más contenidos e interacciones realizaron en Twitter obtuvieron un impacto positivo en el aumento de los votos obtenidos.

En cuanto a la aportación de la presente investigación, está en que no solo se estudia lo que dicen en Twitter unas cuentas u otras y las interacciones con sus audiencias -como en la mayoría de estudios existentes y mencionados-, sino que compara el contenido de las publicaciones con el contenido de los programas electorales de los partidos políticos, para comprobar si dan la misma importancia en ambas plataformas a diferentes te- 
mas recurrentes de campaña electoral. Este tipo de investigación aún no ha sido realizado hasta la fecha en España, donde la mayoría de los estudios relativos al uso de Twitter en política se centran en la personalización de la campaña, en las interacciones o en las estrategias y funciones comunicativas, no en los temas concretos que tratan sus programas electorales y su reflejo en el contenido que crean en Twitter en campaña electoral.

En consecuencia, el presente estudio parte de las siguientes hipótesis: 1) los partidos políticos de nueva creación y sus respectivos candidatos hacen un mayor uso de la red social, lo que se expresa en un mayor volumen de trinos y retrinos durante la campaña electoral; 2) no hay una correspondencia entre los temas del programa electoral de los partidos políticos y su conversación en Twitter; esto es, las cuestiones presentes en dicho programa no tienen la misma presencia en Twitter e incluso desaparecen de la conversación en la red; 3 ) los partidos políticos buscan ideas fuerza para la comunicación -que no el debate- a través de Twitter. Así, los partidos de izquierdas se centrarán más en temas sociales y en los relacionados con la corrupción, mientras que los partidos de derechas se centrarán más en temas económicos y en la defensa de la unidad de España.

\section{Metodología}

La metodología está basada en dos análisis. Por un lado, se emplea el análisis cuantitativo, una técnica que nos permite objetiva y sistemáticamente analizar una gran cantidad de información (Piñuel, 2002; Igartua, 2006), y el análisis cualitativo o de contenido, que se hizo siguiendo las indicaciones de Jungherr (2016) para el diseño de la selección y recolección de los datos en Twitter.

La muestra del análisis cuantitativo la componen los 4.329 mensajes emitidos en la red social Twitter durante la campaña electoral oficial para las elecciones generales del 26 de junio de 2016 por los cuatro principales partidos políticos y los perfiles de sus candidatos a la presidencia. En España, la campaña electoral ocurre en las dos semanas anteriores a la celebración de las elecciones, por lo que los mensajes seleccionados de Twit- 
ter correspondieron a todos los emitidos del 10 de junio al 24 de junio. El 25 de junio, día previo a las elecciones, no se consideró para el de análisis, puesto que la ley lo establece como jornada de reflexión y no se permite la emisión de ningún mensaje político. La elección de estos cuatro partidos se justifica en que juntos superan el $85 \%$ de los votos del país. La muestra compuesta por todos los mensajes publicados en Twitter por las cuentas, tanto de los partidos políticos como de los candidatos a la presidencia, se extrajo usando la herramienta Twitonomy.

El trabajo estadístico de análisis cuantitativo de los 4.329 mensajes extraídos se realizó posteriormente a través del software estadístico SPSS (Statistical Package for the Social Sciences). De esta forma se pretende alcanzar el primer objetivo de la investigación, así como proceder a la verificación de la primera hipótesis. La Tabla 1 muestra el resumen de la actividad analizada, dividida de acuerdo con los partidos políticos y sus candidatos, así como algunas características de los trinos: si contienen o no elementos multimedia, menciones, etiquetas o hashtags y las interacciones en forma de retrinos y respuestas.

Para la segunda parte, se realizó un análisis cualitativo de contenido. Tras haber confeccionado la base de datos con los mensajes de Twitter, se descargaron los programas electorales de los cuatro partidos políticos a analizar y se establecieron sus temas clave. Para descubrir la importancia que cada partido político y sus respectivos candidatos otorgaban a diferentes cuestiones presentes en su programa electoral, se elaboró una lista y se recurrió, además, a la lista de temas electorales elaborada por la Radio y Televisión Española (RTVE), presente en su página web. Finalmente, los temas seleccionados para analizar fueron: empleo, impuestos, economía, energía, reforma constitucional, regeneración democrática, corrupción, sanidad, educación, justicia, asuntos del interior, política social, violencia de género, igualdad, vivienda, terrorismo, inmigración, política exterior, medio ambiente, cultura e $\mathrm{I}+\mathrm{D}$.

Para la selección de dichos temas nos hemos basado en una publicación realizada en la web de RTVE, en la que proponían una serie de temas 
importantes en los programas electorales para comparar las propuestas de diferentes partidos, siendo también los temas sobre los que se preguntó a los candidatos en diferentes entrevistas y debates en la cadena pública. Esta información se sistematizó a través del programa Nvivo, donde cada tema seleccionado constituye un nodo y cada nodo nos permite categorizar y organizar el contenido de todos los trinos analizados.

Tras la selección y análisis de los temas, se comprobó, en primer lugar, el espacio que cada uno de los temas ocupaba en el programa electoral de cada uno de los partidos, estableciendo el porcentaje correspondiente. En la segunda columna, el porcentaje de trinos dedicado a cada tema en concreto, teniendo en cuenta el número total de trinos. En la tercera columna, el porcentaje de trinos dedicado a cada tema en concreto, pero esta vez teniendo solamente en cuenta los trinos que tratasen alguno de los temas del programa electoral. Para ilustrar las diferencias entre partidos y candidatos se adjuntan las tablas que contienen el análisis de los resultados. Cada columna se corresponde con el porcentaje de espacio que ocupa cada cuestión en el programa electoral (1), en el total de trinos publicados (2) y en el total de trinos publicados que aborden las cuestiones que se han propuesto (3).

\section{Análisis de los resultados}

Como punto de partida se analiza el volumen de publicaciones de todos los partidos y de sus candidatos, para poder realizar comparaciones. Exponemos a continuación la distribución de los 4.329 mensajes trabajados:

\section{Tabla 1. Actividad de los Partidos Políticos en Social Twitter, 10-24 de junio de 2016}

\begin{tabular}{|c|c|c|c|c|c|c|c|c|c|c|}
\hline & PP & PSOE & UP & C's & Rajoy & Sánchez & Iglesias & Rivera & Total \\
\hline Publicaciones & 469 & 1.513 & 1.209 & 712 & 165 & 160 & 51 & 50 & 4.329 \\
\hline Retrinos & 6 & 6 & 2 & 1 & 7 & 2 & 3 & 5 & 32 \\
\hline Respuestas & 5 & 0 & 0 & 0 & 1 & 5 & 7 & 4 & 22 \\
\hline Hashtags & 548 & 1.395 & 1.513 & 798 & 281 & 178 & 17 & 40 & 4.770 \\
\hline Menciones & 452 & 1.477 & 1.004 & 690 & 64 & 62 & 31 & 20 & 3.800 \\
\hline Elementos multimedia & 294 & 345 & 973 & 478 & 110 & 163 & 47 & 45 & 2.455 \\
\hline
\end{tabular}

Fuente: elaboración propia. 
Si atendemos al volumen de publicación de las cuentas de los partidos, se puede observar cómo el PSOE fue el que más publicó en su cuenta: más del triple que el último, el PP, seguido de la cuenta de Unidas Podemos, con un total de 1.209 trinos. En tercer lugar, se sitúa la cuenta de Ciudadanos, con 712 trinos y, por último, la cuenta del Partido Popular, con solo 469 trinos. Existe una diferencia muy grande entre el volumen de publicaciones del partido más activo (PSOE) y el menos activo (PP), al que triplica en actividad por esta red social.

La cuenta del PSOE es la que más menciones publicó: en total, 1.412, con Pedro Sánchez (@sanchezcastejon) destacando como personaje más mencionado, con 888 menciones. En este apartado esencialmente cuantitativo de la investigación se ha analizado el uso de elementos multimedia, tales como imágenes, vídeos, GIF, etc. La cuenta del partido que más uso realizó de estos elementos fue Unidas Podemos, con la publicación de 973 elementos multimedia, más del doble que cualquiera de las otras cuentas de Twitter analizadas en la presente investigación. Esta diferencia tan exagerada en este componente puede deberse al público objetivo al que quieren dirigirse, que cuenta con una edad media más baja.

En cuanto al número de hashtags o etiquetas, se aprecia que la cuenta de Unidas Podemos fue la que publicó mayor cantidad y variedad de ellas (1.513), compara con las del resto y triplicando al PP (548), que fue el que menos los ha utilizado. La segunda cuenta que más hashtags publicó fue la del PSOE (1.395) y en tercer lugar la de Ciudadanos, con 798 etiquetas. Cabe destacar que todas las cuentas publicaron mensajes repetidos y algunos fueron recurrentes durante toda la campaña electoral. Es el caso de hashtags como \#26J, utilizado por todos los partidos, o algunos particulares de cada cuenta, como el eslogan \#Afavor, utilizado en varias ocasiones por el Partido Popular.

En cuanto a la actividad de los candidatos, se destaca en primer lugar que, pese a que la cuenta del PP fue la que menos trinos emitió de los cuatro partidos analizados, la de Mariano Rajoy realizó más publicaciones, seguida por la de Pedro Sánchez. Por otro lado, a pesar de que la cuenta del PSOE estuvo más activa publicando, no ocurrió lo mismo con la cuenta de 
su líder. La comparación entre partidos y candidatos según el número de retrinos realizados por sus cuentas muestra que es similar, pero resulta llamativo el número tan bajo de retrinos que todas estas cuentas recibieron.

\section{Figuras 1 y 2 . Actividad de los partidos políticos y de sus candidatos}

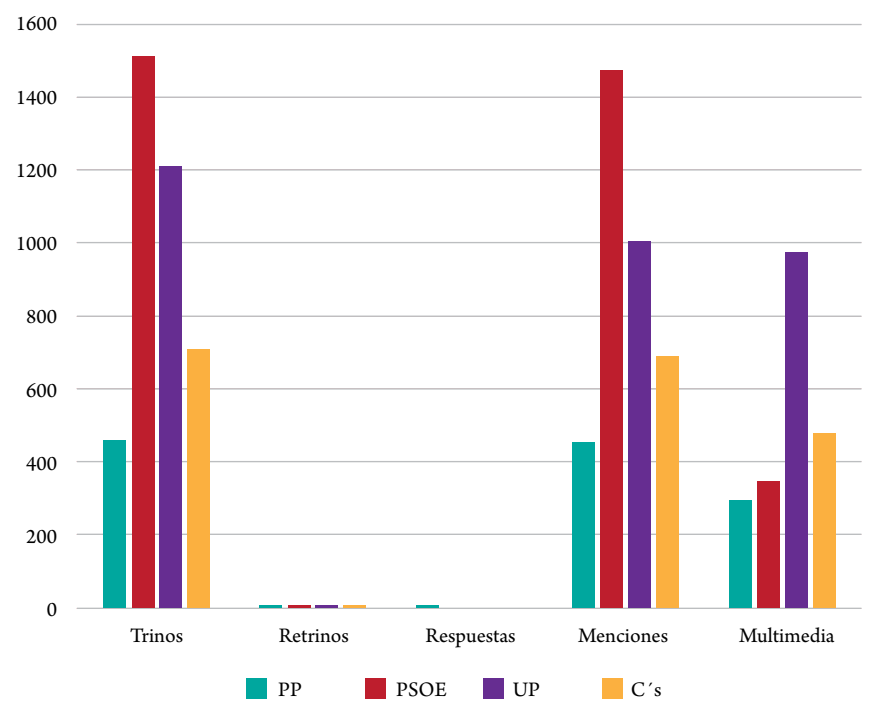

300

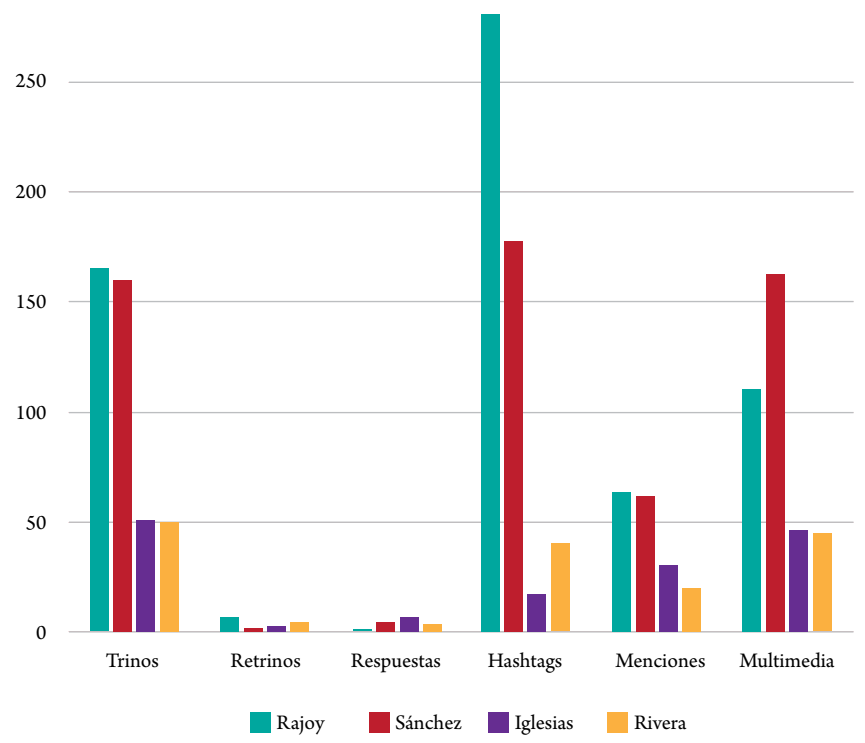

Fuente: elaboración propia. 
El líder que más uso hizo de los elementos multimedia fue Pedro Sánchez (163), seguido de Mariano Rajoy (110), Pablo Iglesias (47) y Albert Rivera (45). En cuanto al número de hashtags publicados destaca, sin duda, Mariano Rajoy, cuyo volumen de publicación de hashtags fue muy superior al del resto de candidatos (281). En segundo lugar, está Pedro Sánchez (178) y en tercer lugar Albert Rivera, con 40 hashtags publicados, mientras en último lugar aparece Pablo Iglesias, con tan solo 17 etiquetas.

\section{Partidos políticos y temas del programa electoral}

Para descubrir la importancia que cada partido político otorgaba a diferentes cuestiones presentes en su programa electoral se elaboró la lista de los temas. Con el fin de ilustrar las diferencias entre partidos y candidatos se elaboraron dos tablas ( 2 y 3 ), en las que cada columna se corresponde con el porcentaje de espacio que ocupa cada cuestión en el programa electoral (1) y en el total de trinos publicados (2) y de trinos que abordaran las cuestiones que se han propuesto (3). El porcentaje de trinos publicados en campaña electoral por cada partido político, teniendo en cuenta el total dedicado a las cuestiones de la lista elaborada con sus programas electorales, es el siguiente: PP: 32,46\%; PSOE: 38,3\%; UP: $21,7 \%$; C's: $25,4 \%$. Se aprecia que el PSOE es el que más contenido publica en Twitter relacionado con los temas que trata en su programa electoral y que Unidas Podemos es el partido que menos equilibrio y concordancia muestra entre los temas que recoge su programa electoral y el contenido de sus mensajes en esta red social.

\section{Tabla 2. Porcentaje de trinos dedicados a las principales cuestiones del programa electoral por los partidos políticos}

\begin{tabular}{|c|c|c|c|c|c|c|c|c|c|c|c|c|}
\hline & \multicolumn{3}{|c|}{ PP } & \multicolumn{3}{c|}{ PSOE } & \multicolumn{3}{c|}{ UP } & \multicolumn{3}{c|}{ C's } \\
\hline & $\mathbf{1}$ & $\mathbf{2}$ & $\mathbf{3}$ & $\mathbf{1}$ & $\mathbf{2}$ & $\mathbf{3}$ & $\mathbf{1}$ & $\mathbf{2}$ & $\mathbf{3}$ & $\mathbf{1}$ & $\mathbf{2}$ & $\mathbf{3}$ \\
\hline Empleo & 2,9 & 12,2 & 37,6 & 3 & 4,1 & 10,7 & 6,6 & 2,7 & 12,5 & 9,1 & 3,9 & 12,1 \\
\hline Impuestos & 2,9 & 1,9 & 6 & 2,5 & 0,8 & 2,1 & 1,6 & 0,8 & 3,8 & 2,3 & 1,8 & 7,2 \\
\hline Economía & 35,1 & 3,7 & 11,4 & 31 & 2,4 & 6,4 & 46,7 & 2 & 9,1 & 15,9 & 4,5 & 17,7 \\
\hline Energía & 2,4 & 0 & 0 & 2,5 & 0,3 & 0,7 & 3,3 & 0,6 & 2,7 & 2,3 & 0 & 0 \\
\hline $\begin{array}{c}\text { Reforma } \\
\text { constitucional }\end{array}$ & 2,9 & 0,2 & 0,7 & 0 & 0,5 & 1 & 1,6 & 0 & 0 & 0,7 & 0,1 & 0,5 \\
\hline
\end{tabular}




\begin{tabular}{|c|c|c|c|c|c|c|c|c|c|c|c|c|}
\hline & \multicolumn{3}{|c|}{$\mathbf{P P}$} & \multicolumn{3}{|c|}{ PSOE } & \multicolumn{3}{|c|}{ UP } & \multicolumn{3}{|c|}{$C^{\prime} s$} \\
\hline & 1 & 2 & 3 & 1 & 2 & 3 & 1 & 2 & 3 & 1 & 2 & 3 \\
\hline $\begin{array}{l}\text { Regeneración } \\
\text { democrática }\end{array}$ & 2,4 & 0,9 & 2,7 & 6,6 & 0,2 & 0,3 & 3,3 & 0 & 0 & 15,9 & 0,6 & 2,2 \\
\hline Corrupción & 2,4 & 2,2 & 6,7 & 1,1 & 1,6 & 4,1 & 3,3 & 3,3 & 15,2 & 11,4 & 2,8 & 11 \\
\hline Sanidad & 4,8 & 0,7 & 2 & 4,9 & 4,4 & 9,1 & 1,6 & 0,6 & 2,7 & 4,5 & 1,3 & 5 \\
\hline Educación & 6,3 & 2 & 6 & 6,6 & 5,1 & 13,3 & 4,9 & 0,7 & 3,4 & 9,1 & 4,3 & 17,1 \\
\hline Justicia & 3,4 & 0 & 0 & 1,9 & 1,1 & 2,8 & 1,6 & 1,1 & 4,9 & 2,3 & 0,3 & 1,1 \\
\hline Interior & 20,2 & 2,2 & 6,7 & 1,4 & 0,5 & 1,2 & 3,3 & 0,7 & 3,4 & 15,1 & 0,6 & 2,2 \\
\hline Política social & 20,7 & 1,5 & 4,7 & 21,1 & 1,3 & 3,3 & 24,6 & 2,1 & 9,9 & 25 & 0,8 & 3,3 \\
\hline $\begin{array}{l}\text { Violencia de } \\
\text { género }\end{array}$ & 2,4 & 0 & 0 & 1,1 & 2 & 5,2 & 3,3 & 0,7 & 3,4 & 2,3 & 0 & 0 \\
\hline Igualdad & 4,8 & 1 & 3,4 & 1,4 & 10,6 & 27,8 & 6,6 & 1,5 & 6,8 & 21,7 & 1,3 & 5 \\
\hline Vivienda & 2,9 & 0 & 0 & 2,7 & 0 & 0 & 4,9 & 0,9 & 4,2 & 1,1 & 0 & 0 \\
\hline Terrorismo & 2,4 & 0,4 & 1,3 & 1,6 & 0,1 & 0,2 & 0 & 0,08 & 0,4 & 2,3 & 0,1 & 0,6 \\
\hline Inmigración & 0,5 & 0,4 & 1,3 & 2,5 & 1,3 & 3,5 & 3,3 & 0,4 & 1,9 & 0,9 & 0 & 0 \\
\hline Política exterior & 9,6 & 3 & 9,4 & 11,8 & 1,3 & 3,3 & 3,3 & 1,7 & 7,7 & 11,4 & 2,8 & 11 \\
\hline Medio ambiente & 1,9 & 0 & 0 & 1,9 & 0,2 & 0,5 & 1,6 & 0,2 & 0,8 & 6,8 & 0 & 0 \\
\hline Cultura & 2,4 & 0 & 0 & 2,5 & 1,3 & 3,5 & 1,6 & 1,2 & 5,7 & 4,6 & 0,6 & 2,2 \\
\hline $\mathrm{I}+\mathrm{D}$ & 4,8 & 0 & 0 & 1,4 & 0,4 & 1 & 3,3 & 0,3 & 1,5 & 6,8 & 0,4 & 1,7 \\
\hline
\end{tabular}

Fuente: elaboración propia.

En primer lugar, se pone la atención en aquellos apartados que ocupan un mayor espacio en el programa electoral de los partidos políticos. El tema al que más espacio del programa le dedicaron todos los partidos políticos ha sido la economía, así: el PP un 35,1\%, el PSOE un 31\%, Unidas Podemos un $46,7 \%$ y Ciudadanos un $15,9 \%$. Un apartado que, debe recordarse, incluye muchas cuestiones. Por ejemplo, dentro del apartado de economía del PP se incluye: empleos para el bienestar de los españoles; reformas para la competitividad; emprendedores y pymes, fundamentales para el desarrollo de nuestro bienestar. Dentro del apartado de economía del PSOE se incluyen los siguientes temas: una economía 4.0 más productiva, un mercado laboral más eficiente, economía verde, sectores, estabilidad presupuestaria, instituciones y mercados inclusivos. Por su parte, en cuanto a Unidas Podemos, partido que más espacio le dedicó al tema económico en su programa, se distinguen los siguientes subapartados: banca 
pública y regulación financiera, sociedad del conocimiento, I+D+i y políticas científicas, agricultura y pesca, sistema de pensiones de calidad, reestructuración de la deuda española, etc. El apartado dedicado a la economía en Ciudadanos es mucho menor que en el resto de los partidos.

Si se atiende ahora al volumen de trinos que tratan el tema económico, se observa que el partido que más publicaciones hizo fue Ciudadanos $(17,7 \%)$, curiosamente el que menos espacio le otorgaba en el programa electoral. El segundo partido que más trinos le dedicó al asunto económico fue el PP (11,4\%), el tercero Unidas Podemos $(9,1 \%)$ y el último el PSOE $(6,4 \%)$. Exceptuando el caso de Ciudadanos, el resto de partidos dedicó mucho más espacio a la economía en el programa que en sus publicaciones.

Otro apartado que ocupó un gran espacio en el programa electoral de los cuatro partidos políticos fue el de política social: al que el PP le dedicó 20,1\%, el PSOE 21,1\%, Unidas Podemos 24,6\% y Ciudadanos $25 \%$. Como se puede observar, pese a ser un partido liberal y de corte conservador, Ciudadanos fue el que más espacio dedicó a la política social, seguido de Unidas Podemos, del PSOE y, por último, del PP. En este caso, ocurre lo contrario que con el apartado de política económica, en el que Ciudadanos es el que menos espacio le dedicó en el programa, cuando en Twitter fue el más activo. Si ahora se tienen en cuenta los trinos publicados sobre el tema, en los porcentajes de la columna 3 se comprueba que el partido que más trinos publicó atendiendo a la política social o temática social fue UP (9,9\%), seguido del PP (4,7\%) y de PSOE y Ciudadanos, ambos con $3,3 \%$ de trinos que incluyeron el tema. En esta ocasión no sucedió lo mismo que con el tema económico, siendo Ciudadanos el que más espacio le dedicó en el programa y el que menos cantidad de trinos emitió.

Otro apartado al que algunos partidos le otorgaron bastante espacio es el de los asuntos del interior. El partido que más espacio le dedicó fue el PP (20,2\%), seguido de Ciudadanos (15,1\%), UP (3,3\%) y PSOE (1,4\%). Cabe señalar que los dos partidos que más espacio le dedicaron incluían los asuntos del interior en un gran apartado, con diferentes subapartados relacionados con la seguridad, la corrupción, la reforma institucional y la 
reforma constitucional. En cuanto al porcentaje de trinos dedicados a este tema, el PP sigue siendo el que más importancia le dio, con un $6,7 \%$ de los trinos dedicados a los asuntos del interior, seguido de UP (3,4\%), Ciudadanos $(2,2 \%)$ y PSOE $(1,2 \%)$.

El partido que más espacio del programa electoral reservó al tema del empleo fue Ciudadanos (9,1\%), seguido de Unidas Podemos (6,6\%), el PSOE (3\%) y el PP (2,9\%). Durante el periodo de campaña todos los partidos abordaron bastante el tema, debido a su trascendencia. Pese a que no se hubo dedicado demasiado espacio en el programa, vemos que, en cambio, Twitter vehiculó un gran porcentaje de posturas expresadas en sus trinos. El partido que mayor porcentaje de trinos realizó sobre empleo fue claramente el PP $(37,6 \%)$, seguido de UP (12,5\%), C's (12,1\%) y PSOE (10,7\%).

En el apartado dedicado a los impuestos podemos ver que el PP es el que más espacio le dedicó en el programa (2,9\%), aunque las diferencias en este apartado son pequeñas, ya que el PSOE le dedicó $2,5 \%$, Ciudadanos 2,3\% y Unidas Podemos 1,6\%. Teniendo en cuenta el porcentaje de trinos publicados acerca del asunto, se aprecia que $C^{\prime} s(7,2 \%)$ es el partido que más importancia le concedió al tema, seguido por poco del PP (6\%), de UP $(3,8 \%)$ y del PSOE $(2,1 \%)$.

Con respecto al apartado del programa electoral dedicado a energía, el partido que más espacio le dedicó fue Unidas Podemos (3,3\%), seguido del Partido Socialista (PSOE) (2,5\%), el Partido Popular (2,4\%) y Ciudadanos $(2,3 \%)$. Las diferencias entre los distintos partidos son mínimas, siendo, además, un tema que tampoco ha estado muy presente en Twitter, lo que indica que los partidos políticos analizados no lo consideraron relevante para esta campaña. Tan solo UP $(2,7 \%)$ y el PSOE $(0,7 \%)$ aludieron en Twitter al asunto de la energía.

En el apartado correspondiente al tema de la corrupción, destaca Ciudadanos en cuanto al espacio ocupado por el tema en el programa (11,4\%), seguido de Unidas Podemos (3,3\%), PP $(2,4 \%)$ y PSOE $(1,1 \%)$. Sin embargo, en cuanto al número de trinos sobre el tema, se aprecian variaciones 
importantes. El partido que más trinó sobre corrupción fue UP (15,2\%), seguido de C's (11\%), del PP (6,4\%) y del PSOE (4,1\%). Tanto UP como C's y PSOE dedicaron gran cantidad de trinos a atacar al PP por los actos de corrupción de muchos de sus integrantes.

Otra de las cuestiones seleccionadas para el análisis es la sanidad. El partido que más espacio le dedicó en su programa electoral fue el PSOE $(4,9 \%)$, seguido del PP $(4,8 \%)$, Ciudadanos $(4,5 \%)$ y UP $(1,6 \%)$. Excepto UP, el resto de partidos le dedicaron casi el mismo espacio. Sin embargo, la cantidad de trinos que unos partidos y otros emitieron en referencia a este tema difiere bastante más. El PSOE sigue siendo el que más relevancia le dio, publicando el $9 \%$ de los trinos acerca de la sanidad, seguido por Ciudadanos con un 5\%, UP con 2,7\% y, por último, el PP con un $2 \%$. Tanto Ciudadanos como el PSOE se centraron en atacar la gestión sanitaria del PP, mientras que UP optó por la defensa en sus trinos del sistema público y universal de sanidad. Por otro lado, el PP atacó al PSOE por "la herencia recibida” en elámbito sanitario y a la vez defendió el sistema público de salud.

Sobre la educación, Ciudadanos es el que más espacio le dedicó en su programa electoral $(9,1 \%)$, seguido del Partido Socialista Obrero Español (6,6\%), el Partido Popular (6,3\%) y Unidas Podemos (4,9\%). En cuanto al volumen de trinos publicados sobre el tema, en este caso, el orden no cambió y C's fue el que más publicó sobre educación $(17,1 \%)$, seguido del PSOE (13,3\%), del PP (6\%) y de UP (3,4\%). En el apartado de justicia, el partido que más espacio se le dio en el programa electoral fue el PP $(3,4 \%)$, seguido de C's $(2,3 \%)$, PSOE $(1,9 \%)$ y UP $(1,6 \%)$. Sin embargo, el porcentaje de publicaciones señala que UP es el partido que más trinó acerca de la justicia $(4,9 \%)$, seguido del PSOE $(1,9 \%)$ y de $C^{\prime}$ s $(1,1 \%)$, mientras que el PP no publicó ningún trino. Aquí se encuentra otro claro ejemplo de que el espacio que ocupan algunas cuestiones en el programa y el volumen de publicaciones sobre el tema en Twitter no coinciden. Los trinos del PSOE y C's contenían críticas al UP, ya que consideraban que, de llegar al poder, querría controlar el sistema judicial. Por otro lado, UP se centró en defender en sus trinos el respeto a las leyes por parte de todos. 
El siguiente apartado que se analiza es el de violencia de género. El partido que más espacio le dedicó al tema en su programa electoral fue Unidas Podemos (3,3\%), seguido del Partido Popular (2,4\%), de Ciudadanos $(2,3 \%)$ y del Partido Socialista $(1,1 \%)$. En cuanto al volumen de publicaciones, solo el PSOE $(5,2 \%)$ y UP $(3,4 \%)$ trinaron sobre el tema, mientras que de los partidos más conservadores no se encontró referencia alguna a la violencia de género en sus trinos. El contenido de las publicaciones del PSOE aportaba básicamente medidas para luchar contra este tipo de violencia, mientras que las publicaciones de UP iban destinadas a condenarla e instaban a combatirla por todos los medios. Medio ambiente y cultura e $\mathrm{I}+\mathrm{D}$ fueron los temas a los que menor presencia otorgaron los partidos $\mathrm{y}$ los candidatos en Twitter.

\section{Candidatos y temas del programa electoral}

En esta sección se hará un análisis individual de cada líder político, comparando su volumen de publicaciones con el de la cuenta de Twitter perteneciente a su partido. Así se descubrirá si los trinos de ambos siguieron una misma dirección o si existen diferencias notables en el tratamiento de determinados temas. En cuanto al contenido de las cuentas oficiales de Twitter de los candidatos a la presidencia del gobierno, el líder que más contenido publicó coincidente con los temas que proponían sus programas electorales fue Albert Rivera, seguido de Pedro Sánchez, ya que ambos en más del $30 \%$ de sus publicaciones trataron alguno de los temas que recogían sus propuestas políticas partidistas. El candidato que menos concordancia mostró fue Pablo Iglesias. En cuanto al porcentaje de contenido publicado en Twitter por cada candidato coincidente con los temas que movían sus programas electorales, se distribuye así: Rajoy: 23,6\%, Sánchez: 30,63\%, Iglesias: $11,76 \%$, Rivera: $32 \%$.

Respecto a los temas relacionados con la cuestión económica, Rajoy le dedicó el 5,1\% de sus trinos, mientras que la cuenta de su partido más que lo duplicó $(11,4 \%)$. En el apartado de igualdad, Rajoy también trató bastante el tema, estando presente en el $12,8 \%$ de sus trinos, pero en la cuenta del partido la igualdad solo estuvo presente en el 3,4\% de sus publicaciones. En el tema del empleo se podría afirmar que ambas cuentas 
coincidían, y aunque en algunos apartados sí se aprecian equivalencias en cuanto al tratamiento de ciertos temas, generalmente las publicaciones de partido y candidato no siguieron una misma línea, con un número de disparidades mayor que el de coincidencias.

Los temas a los que la cuenta de Pedro Sánchez no aludió fueron: impuestos, energía, reforma constitucional, regeneración democrática, vivienda, terrorismo, medio ambiente, cultura e I+D. En la cuenta del partido tampoco se trinó sobre vivienda, pero sí sobre el resto, aunque fuese mínimamente. No obstante, Sánchez fue el único líder, además, que trató los temas de sanidad y violencia de género en sus mensajes en Twitter.

La cuenta de Unidas Podemos y la de su líder siguieron caminos totalmente diferentes, al menos en lo que a información sobre temas del programa electoral se refiere, $y$, como se observa en la Tabla 3, fueron muchos los asuntos sobre los que el candidato de Unidas Podemos no trinó, centrándose casi en solo cuatro temas: regeneración democrática, política social, igualdad y política exterior. Rivera fue el único líder que superó a su partido en cuanto a las cuestiones electorales propuestas, centrado sobre todo en política exterior, igualdad, educación y corrupción. Y fue el único líder en tratar el tema del I+D.

\section{Tabla 3. Porcentaje de trinos dedicados por cada candidato a las principales cuestiones del programa electoral}

\begin{tabular}{|c|c|c|c|c|}
\hline & Rajoy & Sánchez & Iglesias & Ribera \\
\hline Empleo & 0 & 8,2 & 0 & 6,2 \\
\hline Impuestos & 0 & 0 & 0 & 0 \\
\hline Economía & 5,1 & 4,1 & 0 & 0 \\
\hline Energía & 0 & 0 & 0 & 0 \\
\hline Reforma constitucional & 0 & 0 & 0 & 0 \\
\hline Regeneración democrática & 0 & 0 & 16,7 & 6,2 \\
\hline Corrupción & 5,1 & 4,1 & 0 & 12,5 \\
\hline Sanidad & 0 & 12,2 & 0 & 0 \\
\hline
\end{tabular}




\begin{tabular}{|c|c|c|c|c|}
\hline & Rajoy & Sánchez & Iglesias & Ribera \\
\hline Educación & 7,7 & 14,3 & 0 & 12,5 \\
\hline Justicia & 0 & 8,2 & 0 & 6,2 \\
\hline Interior & 0 & 8,2 & 33,3 & 0 \\
\hline Política social & 2,6 & 4 & 0 & 0 \\
\hline Violencia de género & 0 & 6,1 & 16,7 & 0 \\
\hline Igualdad & 12,8 & 14,3 & 0 & 6,2 \\
\hline Vivienda & 0 & 0 & 0 & 0 \\
\hline Terrorismo & 7,7 & 0 & 0 & 18,7 \\
\hline Inmigración & 2,6 & 4,1 & 33,3 & 0 \\
\hline Política exterior & 20,5 & 12,2 & 0 & 0 \\
\hline Medio ambiente & 0 & 0 & 0 & 12,5 \\
\hline Cultura & 0 & 0 & 0 & 0 \\
\hline I+D & & 0 & 0 & 0 \\
\hline
\end{tabular}

Fuente: elaboración propia.

\section{Conclusiones y discusión}

Atendiendo a los datos recogidos durante la presente investigación, ya se puede afirmar que los partidos políticos de nueva creación y sus respectivos candidatos no hicieron un uso amplio de la red social Twitter, lo cual se expresó en el volumen de trinos y retrinos durante la campaña electoral. apreciación consistente con el hecho de que fuera la cuenta del PSOE - partido fundado hace más de cien años- la que más trinos publicó durante el periodo de campaña electoral (1.513 trinos en total). Por otro lado, atendiendo al volumen de publicación de trinos por los cuatro candidatos, observamos que la cuenta de Mariano Rajoy fue la más activa (165 trinos), como líder del Partido Popular, de amplia trayectoria en España. Por lo tanto, se concluye que la modernidad adjudicada a los partidos de nueva creación no redunda en una mayor actividad en la red social Twitter.

Por otro lado, llama la atención la disparidad entre el volumen de publicación del Partido Popular, que se posiciona en último lugar en este sentido, y el de su candidato, Mariano Rajoy, que se presenta como el más activo en cuanto a trinos publicados. También existen diferencias significativas en- 
tre los líderes de los partidos tradicionales y los líderes de los partidos más nuevos en otras cuestiones, como el uso de elementos multimedia, visto que, curiosamente, estos últimos fueron los que menos recurrieron a ellos. Es bastante llamativo que los dos partidos de nueva creación hayan publicado un número tan bajo de etiquetas o hashtags, sobre todo en el caso del líder de Unidas Podemos, ya que su uso puede hacer más visibles algunas publicaciones y provocar que estas puedan llegar a un número mayor de personas. Aunque, por otro lado, las publicaciones de Iglesias ya suelen alcanzar altos porcentajes de viralidad, debido a que era y es el líder más seguido y cuenta con una diferencia de seguidores muy amplia con respecto al resto de candidatos. Unidas Podemos es el partido que posee un mayor número de seguidores (1.330.000 de seguidores), pero no fue el que más publicó en su cuenta de Twitter, sino el segundo (1.209 trinos), y su candidato Pablo Iglesias quedó como segundo con menos trinos (51), solo por delante de Albert Rivera (50 trinos). En contra de la percepción previa a esta investigación, los dos candidatos de los partidos de más reciente creación fueron los que menos publicaron, algo más llamativo en el caso de Iglesias, habida cuenta de que es el candidato con más seguidores $(2.230 .000$ de seguidores).

Además, cabe destacar que las tendencias de uso en la red social han cambiado. Como concluyeron Castillejo y Sémova (2012) en su investigación, el uso de Twitter hecho por el PP y el PSOE en 2011, en general, fue para informar sobre actividades electorales y para retuitear información. En la presente investigación resulta llamativo el número tan bajo de retrinos que todas estas cuentas realizaron, por lo que vemos claramente un cambio de tendencia en el uso político de Twitter en España, donde ya no se retuitea casi y se crean nuevos mensajes en campaña electoral, muchos de ellos, un 56,7\% concretamente, contienen elementos multimedia: fotos, vídeos, GIF, etc.

En cuanto a la hipótesis que planteaba una correspondencia entre los temas del programa electoral de los partidos políticos y su conversación en Twitter, se probó que un $15 \%$ de los temas recogidos en el programa electoral ni siquiera fueron mencionados por los partidos en sus cuentas de 
Twitter y otro $15 \%$ fueron mencionados únicamente por dos partidos y de manera proporcional a su presencia en el programa. Además, la gran mayoría de los temas recogidos en los programas electorales, el $70 \%$, fueron publicados en Twitter únicamente por un partido de manera proporcional al espacio dedicado en el programa electoral, con una clara diferenciación entre las cuestiones en las que centraron sus mensajes los partidos más cercanos a la izquierda o a la derecha. Cabe destacar que la economía estuvo muy presente en los programas pero, sorprendentemente, no tanto en las cuentas oficiales de los partidos. Se afirma, por tanto, que los partidos no diseñan su estrategia comunicativa en Twitter atendiendo a sus programas electorales, algo que no solo confirma la segunda hipótesis, sino que apunta, quizá, a una falta de comunicación estratégica integrada en periodos electorales o a la aún pendiente inclusión de la utilización estratégica de Twitter en el plan de comunicación diseñado para cada campaña electoral.

Como hallazgo adicional, los partidos y sus candidatos no publican sobre los mismos asuntos y, si lo hacen, en absoluto es en la misma medida. El único partido que trata de lograr una mínima coherencia entre los mensajes de partido y candidato es el PSOE, algo que no ocurre en el resto de candidatos y partidos analizados. El PSOE y Pedro Sánchez fueron los que más alineadas llevaban las estrategias de creación de contenido en Twitter en relación con los temas que trataba su programa electoral. En este sentido, se puede vincular esta falta de coherencia con una de las conclusiones que se apuntaban tras la confirmación de la segunda hipótesis, referente a la falta de coordinación de la comunicación de los partidos con respecto a los programas electorales y la red social estudiada, y también con respecto a la comunicación diseñada para los líderes de cada uno de los partidos.

La tercera hipótesis también fue validada, pues se vio que los partidos políticos buscan ideas fuerza para la comunicación -que no debate-a través de Twitter. Así, los de izquierda se centran más en temas sociales y en asuntos relacionados con la corrupción, mientras que los de derecha se enfocan más en temas económicos y en la defensa de la unidad de España. El PP, partido de derecha, dedicó un mayor número de trinos a empleo $(37,6 \%)$, economía $(11,4 \%)$ y política exterior $(9,4 \%)$, y no mencionó siquiera cues- 
tiones más sociales sobre, justicia, violencia de género, vivienda, medio ambiente, cultura e I+D o energía. El PSOE, partido de izquierda, dedicó un mayor número de trinos a igualdad (27,8\%), educación (13,3\%) y empleo $(10,7 \%)$, dejando de lado temas como vivienda $(0 \%)$, terrorismo $(0,2 \%)$ y regeneración democrática $(0,3 \%)$. UP, también partido de izquierda, dedicó un mayor número de trinos a la corrupción $(15,2 \%)$, empleo $(12,5 \%)$ y política social $(9,9 \%)$, pero sin mencionar la reforma constitucional $(0 \%)$, la regeneración democrática $(0 \%)$ y apenas tampoco el terrorismo $(0,4 \%)$. Por último, Ciudadanos dedicó un mayor número de trinos a la economía $(17,7 \%)$, educación $(17,1 \%)$ y empleo $(12,1 \%)$, sin mencionar cuestiones como energía, vivienda, violencia de género y medio ambiente.

Finalmente, tras la realización de esta investigación, se considera que, por un lado, la intensidad con la que tanto candidatos como partidos políticos emplearon Twitter durante la campaña electoral confirma la consolidación de esta red social como herramienta para la comunicación electoral en España. Sin embargo, en esta campaña en concreto la falta de coherencia entre los temas abordados en los trinos de partidos y candidatos, así como la ausencia de proporcionalidad en la importancia otorgada a las distintas cuestiones en el programa electoral y en la "conversación" dentro de la red social, apuntan a que Twitter no ha estado incorporada como herramienta de comunicación a la comunicación integral de candidatos y partidos para propagar su programa electoral. López, Marcos y Casero (2017) estudiaron también las elecciones de 2016 en España, tanto durante la campaña electoral como el mismo día y el posterior de las votaciones por los cuatro partidos más votados y sus candidatos. Los resultados de su estudio coinciden con los de esta investigación y mostraron, por un lado, que Pablo Iglesias fue el candidato que menos contenido publicó en Twitter coincidente con el que recogía el programa electoral de su partido político, y, por otro, que PP y PSOE usaron Twitter para informar de sus propuestas políticas y de su programa electoral (así como su líder Pedro Sánchez) dedicando una tercera parte de sus publicaciones a esta función.

¿Entienden Twitter los partidos políticos como un canal de comunicación a través del cual es posible hacer llegar su programa electoral a sus 
audiencias? Damos una respuesta negativa a esta cuestión, añadiendo que en la mayoría de los casos obvian su naturaleza interactiva, utilizando el canal de manera unidireccional. Resultaría de interés ahondar en si esta falta de integración de Twitter como herramienta de comunicación para la difusión del programa electoral obedece a una estrategia previamente establecida por los candidatos y los partidos o simplemente responde a una ausencia de estrategia de comunicación.

Los datos de actividad en Twitter van en descenso en los últimos años, según diferentes estudios, y en cambio los datos en actividad y número de usuarios de otras redes, como Instagram, registran unos ascensos muy llamativos. Selva y Caro (2017) señalan en su estudio que cabría esperar que Instagram se erija en una red social con grandes posibilidades para la comunicación política, por lo que futuras investigaciones podrían abordar el uso que los partidos políticos y sus candidatos empiecen a hacer de dicha red social.

\section{Referencias}

Alonso Muñoz, L., Miquel Segarra, S. y Casero Ripollés, A. (2016). Un potencial comunicativo desaprovechado. Twitter como mecanismo generador de diálogo en campaña electoral. Obra Digital, 11, 3958. DOI: https://doi.org/10.25029/od.2016.100.11

Anduiza Perea, E. (2009). Internet, campanyes electorals i ciutadans. Quaderns del CAC, 33, 5-12. https://dialnet.unirioja.es/servlet/articulo? codigo $=3123645$

Broersma, M. y Graham, T. (2013). Twitter as a news source. Journalism Practice, 7(4), 446-464. DOI: https://doi.org/10.1080/175127 86.2013.802481

Campos-Domínguez, E. (2017). Twitter y la comunicación política. El Profesional de la Información, 26(5), 785-793. DOI: https://doi. org/10.3145/epi.2017.sep.01 
Castillejo, B. y Semova, D. (2012). Elecciones generales y redes sociales en el caso de España, 2011. International Review of Information Ethics, 18, 144-149. DOI: https://doi.org/10.29173/irie312

Congosto, M. L. (2015). European elections 2014: Virality of messages on Twitter. Redes. Revista Hispana para el Análisis de Redes Sociales, 26(1), 23. DOI: https://doi.org/10.5565/rev/redes.529

Congosto, M.L., Fernández, M.y Moro, E. (2011). Twitter y política: información, opinión y ipredicción? Cuadernos de Comunicación Evoca, 4, 11-16. http://evocaimagen.com/cuadernos/cuadernos4.pdf

Conway, B., Kenski, K. y Wang, D. (2013). Use by presidential primary candidates during the 2012 campaign. American Behavioral Scientist, 57(11), 1596-1610. DOI: https://doi. org/10.1177/0002764213489014

Dader, J. L. (2009). Ciberpolítica en los websites de partidos políticos. La experiencia de las elecciones de 2008 en España entre las tendencias transnacionales. Revista de Sociología e Política, 17(34). DOI: https://doi.org/10.1590/S0104-44782009000300005

Elorriaga Illera, A., Merchan Mota, I. y Vink Larruskain, N. (2018). El Social Big Data: una oportunidad empresarial y laboral. Estudios sobre el Mensaje Periodístico, 24(2), 1213-1222. DOI: https://doi. org/10.5209/ESMP.62210

García Ortega, C. y Zugasti Azagra, R. (2014). La campaña virtual en Twitter: análisis de las cuentas de Rajoy y de Rubalcaba en las elecciones generales de 2011. Historia y Comunicación Social, 19, 299-311. DOI: https://doi.org/10.5209/rev_HICS.2014.v19.45029

Giansante, G. (2015). La comunicación política online. Barcelona: UOC.

Gibson, R. y Römmele, A. (2008). Political Communication. En Comparative politics (pp. 473-489). Oxford: Oxford University Press. 
Hendricks, J. A. y Denton, R. E. (eds.) (2010). Communicator-in-chief: how Barack Obama used new media technology to win the White House. Lanham: Lexington Books.

Igartua, J. J. (2006). Métodos cuantitativos de investigación en comunicación. Barcelona: Bosch.

Iturregui, L., Gutiérrez, J. J. y Cantalapiedra, M. J. (2020). Periodistas y profesionales de Relaciones Públicas: de la influencia yla dependencia a la desintermediación periodística. El Profesional de la Información, 29(3). Recuperado de: https://revista.profesionaldelainformacion.com/index.php/EPI/article/view/75623/

Jackson, N. y Lilleker, D. (2011). Microblogging, constituency service and impression management: UKMPs and the use of Twitter. The Journal of Legislative Studies, 17(1), 86-105. DOI: https://doi.org/10. 1080/13572334.2011.545181

Jorge, J. E. (2014). La comunicación política en las redes sociales. Revista Question, 1(44),268-286. Recuperado de: http://sedici.unlp.edu. ar/handle/10915/43802

Jungherr, A. (2016). Twitter use in election campaigns: A systematic literature review. Journal of Information Technology \& Politics, 13(1), 72-91, DOI: https://doi.org/10.1080/19331681.2015.1132401

Kruikemeier, S. (2014). How political candidates use Twitter and the impact on votes. Computers in Human Behavior, 34, 131-139. DOI: https://doi.org/10.1016/j.chb.2014.01.025

López García, G., Gamir Ríos, J. V., García Ull, F. J., Llorca Abad, G., Cano Orón, L., González Esteban, J. L. (2015). El debate sobre Europa en Twitter. Discursos y estrategias de los candidatos de las elecciones al Parlamento Europeo de 2014 en España. Revista de Estudios Políticos, 170,213-246. DOI: https://doi.org/10.18042/cepc/rep.170.07 
López Meri, A. et al. (2016). Twitter-retórica para captar votos en campaña electoral. El caso de las elecciones de Cataluña de 2015. https:// comunicacionyhombre.com/article/twitter-retorica-captar-votos-campana-electoral-caso-las-elecciones-cataluna-2015/

López Meri, A., Marcos-García, S., Casero-Ripollés, A. (2017). What do politicians do on Twitter? Functions and communication strategies in the Spanish electoral campaign of 2016. El Profesional de la Información, 26(5), 795-804. DOI: https://doi.org/10.3145/ epi.2017.sep.02

Mancera Rueda, A. y Pano Alamán, A. (2014). Nuevas dinámicas discursivas en la comunicación política en Twitter. Círculo de Lingüística Aplicada a la Comunicación, 56(0). DOI: https://doi.org/10.5209/ rev_CLAC.2013.v56.43867

Orbegozo Terradillos, J., Iturbe Tolosa, A. y González Abrisketa, M. (2017). Análisis de la nueva estrategia comunicativa de EH Bildu (2016): hacia una narrativa de la emoción. Anàlisi. Quaderns de Comunicació i Cultura, 57, 97-114. DOI: https://doi.org/10.5565/rev/ analisi.3111

Pérez, J. A., Peña, S., Genaut, A., Iturregui, L. y Mendiguren, T. (2013). Comunicación política e Internet: Estrategias online de los partidos políticos vascos en las elecciones autonómicas de 2009. Mediati$k a, 14,125-150$.

Peytibi, F. X., Rodríguez, J. A. y Gutiérrez-Rubí, A. (2008). La experiencia de las elecciones generales del 2008. IDP. Revista de Internet, Derecho y Política, 7. DOI: https://doi.org/10.7238/idp.v0i7.495

Piñuel-Raigada, J. L. (2002). Epistemología, metodología y técnicas del análisis de contenido. Estudios de Sociolingüística, 3(1), 1-42. 
Ruiz-Soler, J. (2017). Twitter research for social scientists: A brief introduction to the benefits, limitations and tools for analysing Twitter data. Revista Dígitos, 1(3), 17-32.

Sampietro, A. y Valera Ordaz, L. (2015). Emotional politics on Facebook. An exploratory study of Podemos' discourse during the European election campaign 2014. Recerca. Revista de Pensament $i$ Anàlisi, 17, 61-83. DOI: https://doi.org/10.6035/Recerca.2015.17.4

Selva-Ruiz, D. y Caro-Castaño, L. (2017). Uso de Instagram como medio de comunicación política por parte de los diputados españoles: la estrategia de humanización en la "vieja" y la "nueva" política. El Profesional de la Información, 26(5), 903-915. DOI: https://doi. org/10.3145/epi.2017.sep.12 